\title{
PRAMOTE STORAGE
}

JANET, Charles

\section{SUR LA MORPHOLOGIE DES MEMBRANES}

\section{BASALES DE L'INSECTE}

Extrait des Memoires de la Societé Academique de l'Oise. - Année 1909.

\section{BEAUVAIS}

IMPRIMERIE DÉPARTEMENTALE DE L'OISE

15, rue des Flageots 


\section{SUR LA MORPHOLOGIE DES MEMBRANES BASALES DE L'IISSECTE}

A la suite du travail intitulé : Sur la Morphologie de l'Insecte, qui constitue le vingt-septième fascicule de mes études sur l'Embranchement des Arthropodes, et dont j'offre un exemplaire à la Société, je ferai paraittre, et cela très prochainement, une étude intitulée : Sur l'Ontogénie de l'Insecle. Enfin, dans un troisième travail actuellement en cours et qui sera peut-être intitulé : Vues théoriques sur la Phylogénie de l'Insecte, j'exposerai ma manière de voir sur l'Evolution ancestrale de la Classe, depuis le Protozoaire jusqu'à l'Insecte supérieur actuel représenté, par exemple, par la Fourmi.

Dans ce travail seront examinés successivement les stades phylogénétiques suivants :

Colonie sphérique de Protozoaires, Blastea, Depea, Gastrea, Siphonea simple, Triade d'individus simples et, enfin, Chaîne annélidienne résultant de l'assemblage de triades.

Dans une coupe transversale de l'abdomen, la basale méso. dermique tégumentaire est en continuité avec la basale mésodermique diaphragmatique. Si l'on part de la ligne sagittale sternale, sur l'épiderme, pour arriver à la ligne sagittale sur le diaphragme sternal, la continuité s'établit comme suit :

$1^{\circ}$ Basale épidermique sternale;

$2^{\circ}$ Basale épidermique tergale;

$3^{\circ}$ Ligaments distaux de l'hémicarde;

$4^{\circ}$ Basale de l'hémicarde (face interne du cueur);

$5^{\circ}$ Ligaments proximaux de l'hémicarde;

$6^{\circ}$ Basale diaphragmatique tergale ou des muscles aliformes ;

$7^{\circ}$ Contact avec la basale épidermique, sur la ligne tégumentaire sterno-tergale;

8. Basale diaphragmatique sternale. 
3 SUR LA MORPHOLOGIE DES MEMBRANES BASALES DE L'INSECTE

La réflexion, par invaginations stomentérique et proctentérique, des basales tégumentaires sternale et tergale donne la basale proximale de la musculature du tube digestif, et la réflexion des basales diaphragmatiques sternale et tergale donne la basale distale de celte musculature.

Ces basales limitent:

1. Par leurs faces musculaires :

a) Une cavité schizocoelienne, intra-mésodermique, sternale (sinus sternal, contenant la chaine des centres nerveux),

b) Une cavité schizocoelienne, intra-mésodermique, tergo-latérale, séparée de sa symétrique par le coeur (sinus tergal),

c) Une cavité schizocoelienne, intra-mésodermique. extrêmement réduite, contenant la musculeuse du tube digestif;

2. Par leurs faces opposées aux faces musculaires:

a) La cavité du coeur,

b) Le coelome.

Il résulte de cette description que, si l'on part du centre du tube digestif pour aller vers le tégument, on rencontre successivement :

$1^{\circ}$ L'épithelium du tube digestif;

$2^{\circ}$ La basale proximale de la musculeuse du tube digestif ;

3. La musculeuse, logée dans une cavilé schizocoelienne;

$4^{\circ} \mathrm{La}$ basale dislale de la musculeuse du tube digestif;

$5^{\circ}$ Le coelome;

$6^{\circ}$ La basale diaphragmatique;

$7^{\circ}$ Son revètement musculaire;

$8^{\circ}$ Le schizocoele intra-mésodermique;

$9^{\circ}$ La musculature tégumentaire;

$10^{\circ} \mathrm{La}$ basale accolée à l'épiderme;

$11^{\circ}$ L'épiderme.

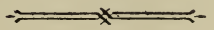


